\title{
31. PETROGRAPHY OF OPAQUE MINERALS IN BASALTS DRILLED ON DSDP LEG 45
}

\author{
P. Eisenach, Institut fur Allgemeine und Angewandte Geologie, Ludwig-Maximilians Universität, München, \\ Federal Republic of Germany
}

\section{INTRODUCTION}

Basalts have been studied for centuries, but intensive studies of the opaque mineral phases they contain, especially of the iron-titanium oxides, have only recently been undertaken. These, even if they constitute less than 1 volume per cent of the whole, are the carriers of the natural remanent magnetization (NRM). The contribution of ilmenite and sulfides to the magnetization can be neglected. Reference to the ternary system $\mathrm{FeO}-\mathrm{Fe}_{2} \mathrm{O}_{3}-\mathrm{TiO}_{2}$ is necessary for interpretation of palaeomagnetism data. Different oxidation states, high temperature, deuteric oxidation (classified in six stages of increasing oxidation [Watkins, 1967]), lowtemperature oxidation $\left(\mathrm{T}<360^{\circ} \mathrm{C}\right)$ and, above all, doping cations $-\mathrm{Mg}, \mathrm{Al}$, and subordinate $\mathrm{Mn}$-produce a wide range of mineralogic and magnetic changes. To study all these phenomena in submarine basalts, investigated so far mainly in subaerial basalts, also for submarine basalts, was the aim of this work.

Three samples of pillow basalt from Hole 395, 25 samples of coarser grained basalt and an intrusive dolerite dike from Hole 395A, and 19 samples of pillow basalt from Hole 396 were examined under reflected light. On five selected coarser grained samples, $200 \mathrm{mi}-$ croprobe analyses of iron-titanium oxides were performed to determine the different oxidation states, especially to estimate low-temperature oxidation and to specify $\mathrm{f}\left(\mathrm{O}_{2}\right)$-T conditions (Buddington and Lindsley, 1964) for the coexisting titanomagnetite-ilmenite phases.

In this work I attempt finally to elucidate the crystallization history and the alteration stage of the irontitanium oxides.

\section{EXPERIMENTAL METHODS}

Polished sections of the samples were examined in reflected light with a Leitz Ortholux Pol microscope. To aid identification of the magnetic minerals and their variable stages of maghemitization, magnetic colloids have also been used. Detailed chemical information on the iron-titanium oxides was obtained at the University of Lausanne-Dorigny, using an ARL electron probe microanalyzer.

\section{RESULTS}

A description of each sample and the average grain sizes of opaque minerals are presented in Plates 1 through 4 and Table 1 . The plates illustrate typical textures and different types of intergrowths of the opaque phases. The chemical data of the iron-titanium oxides and the $\mathrm{f}\left(\mathrm{O}_{2}\right)$-T conditions, using the data of Buddington and Lindsley (1964), are listed in Table 2.

\section{TEXTURES}

\section{Hole 395}

Small skeletal to anhedral grains of titanomagnetite form the bulk of opaque minerals. These grains are uniform in texture and alteration throughout the basalts. Under high magnification, one frequently can see reddish internal reflections. Larger grains show volume-change cracks, a typical sign of low-temperature oxidation.

Primary ilmenite has an elongate shape, and is occasionally mantled by titanomagnetite. Variable proportions of tiny, usually roundish grains of sulfides occur in all rocks. Microphenocrysts of porous chromium spinel occur in Samples 18-2, 31-33 cm and 18-2, 35-37 $\mathrm{cm}$ always show small seams of titanomagnetite. Homogeneous chromium spinels surrounded by pyroxene or plagioclase phenocrysts are not rimmed by titanomagnetite (Plate 1, Figures 1, 2).

\section{Hole 395A}

\section{Titanomagnetite}

Titanomagnetite (with a high content of $\mathrm{TiO}_{2}$ ) is present in all basalt samples. It is usually crystallized in skeletal to anhedral forms indicating a history of rapid cooling of the rocks. In the marginal zones of the pillows and at the top or at the base of thicker flows, one can see a tendency to anhedral forms, resulting from rapid cooling. In the slightly coarser grained basalts, the evolution of well-developed skeletal forms to larger subhedral forms continues. Besides the variation of grain shape and size in the more massive basalts, two size generations of titanomagnetite occur, a larger one (up to $150 \mu \mathrm{m}$ grain size) with usually skeletal to subhedral forms, and a smaller one with skeletal to anhedral forms from $10 \mu \mathrm{m}$ down to the limit of visibility. The smaller titanomagnetites lie near the groundmass pyroxenes, which are generally dendritic, whereas the larger titanomagnetites occur near larger phenocrysts. Observed under oil, stoichiometric titanomagnetites are a homogeneous medium brown. Grains with this appearance are very rare in these rocks, and the bulk of them show varying stages of alteration of what one presumes were original stoichiometric titanomagnetites. Reddish internal reflections, widespread mottled color, and volume-change cracks are typical signs of low-temperature oxidation in all samples-a common phenomenon of submarine basalt rocks (Petersen et al., in press; Ade-Hall and Johnson, 1976). In the volcanic 
TABLE 1

Opaque Minerals of Holes 395, 395A, and Site 396 Basalt

\begin{tabular}{|c|c|c|c|c|c|}
\hline $\begin{array}{c}\text { Sample } \\
\text { (Interval in } \\
\text { cm) }\end{array}$ & Lithology & Titanomagnetite & Ilmenite & Sulfides & Other Phases \\
\hline $11-2,100-106$ & $\begin{array}{l}\text { fine-grained } \\
\text { aphy. basalt }\end{array}$ & $\begin{array}{l}\text { Two generations; tiny skeletal to anhedral } \\
\text { forms, partly as a rim round the plag.laths. } \\
\text { The larger skeletal ones contain expansion } \\
\text { cracks and are fringed by secondary titan- } \\
\text { omagnetite; weak anisotropic; } \\
\text { smaller: } 1 \mu \mathrm{m} \text {-sub. } \\
\text { larger: } 50 \mu \mathrm{m}-10 \mu \mathrm{m}\end{array}$ & $\begin{array}{l}\text { Small primary laths often mantled } \\
\text { by titanomagnetite; } 10-5 \mu \mathrm{m} \text {. }\end{array}$ & $\begin{array}{l}\text { Masses of small grains of pyrrhotite, } \\
\text { especially near the iron-titanium } \\
\text { oxides; } 10 \mu \mathrm{m} \text {-sub. }\end{array}$ & A large euhedral porous chromite \\
\hline $18-2,31-33$ & $\begin{array}{l}\text { pl.ol. } \\
\text { phyric basalt }\end{array}$ & $\begin{array}{l}\text { Only tiny skeletal grains (snowstars) } \\
\text { showing reddish internal reflections under } \\
\text { highest magnification; } \\
10 \mu \mathrm{m} \text {-sub. }\end{array}$ & $\begin{array}{l}\text { Rare small primary laths; } \\
10 \mu \mathrm{m} \text {-sub. }\end{array}$ & $\begin{array}{l}\text { Extremely fine roundish indet. } \\
\text { grains; } 1 \mu \mathrm{m}\end{array}$ & $\begin{array}{l}\text { A large euhedral porous chromite } \\
\text { grain, slightly mottled and } \\
\text { rimmed by a tiny seam of titano- } \\
\text { magnetite; } 50 \mu \mathrm{m}\end{array}$ \\
\hline $18 \cdot 2,35-37$ & $\begin{array}{l}\text { pl.ol.cpx. } \\
\text { phyric basalt }\end{array}$ & Like Sample 18-2, $31-33 \mathrm{~cm}$. & & & $\begin{array}{l}\text { A large euhedral porous grain, } \\
\text { partly rimmed by titanomagnet- } \\
\text { ite and homogeneous ones sur- } \\
\text { rounded by pyroxene and/or } \\
\text { plag.phenocrysts; } 70 \mu \mathrm{m}\end{array}$ \\
\hline $8 \cdot 1,127-135$ & aph. & $\begin{array}{l}\text { Subhedral to anhedral forms; grain size } \\
\text { down to the limit of visibility; some } \\
\text { fringed by a fine titanomag., some show- } \\
\text { ing red staining around the grains; } 1 \mu \mathrm{m} \text { - } \\
\text { sub. }\end{array}$ & $\begin{array}{l}\text { Some tiny laths and rare larger } \\
\text { grains mantled by titanomagne- } \\
\text { tite; } 10 \mu \mathrm{m} \text {-sub. }\end{array}$ & $\begin{array}{l}\text { Extremely fine grains, too small for } \\
\text { det. } 1 \mu \mathrm{m}\end{array}$ & \\
\hline $11-1,56-66$ & aph. & $\begin{array}{l}\text { Subhedral to skeletal shapes, which are } \\
\text { contained particular in the roundish } \\
\text { areas of the groundmass; } 20 \mu \mathrm{m} \text {-sub. }\end{array}$ & $\begin{array}{l}\text { Small primary laths sometimes } \\
\text { mantled by titanomag.; } 5 \mu \mathrm{m} \text {-sub. }\end{array}$ & $\begin{array}{l}\text { Rare extremely fine indet. grains; } \\
1 \mu \mathrm{m}\end{array}$ & \\
\hline $14-1,87-99$ & pl.ol.ph. & $\begin{array}{l}\text { Two size generations of skeletal to sub- } \\
\text { hedral forms; expansion crack and } \\
\text { mottling; partly larger ones fringed by } \\
\text { titanomag.; tiny white reflecting parti- } \\
\text { cles may be hematite; } \\
\text { larger: } 60 \mu \mathrm{m} \\
\text { smaller: } 1 \mu \mathrm{m}\end{array}$ & $\begin{array}{l}\text { Increased content of small pri- } \\
\text { mary laths, partly arranged in a } \\
\text { parallel direction; } 30 \mu \mathrm{m}\end{array}$ & $\begin{array}{l}\text { Rare small pyrrhotite and finer } \\
\text { indet. grains; } 1 \mu \mathrm{m}\end{array}$ & Rare content of iron hydroxide \\
\hline $14-2,125-134$ & pl.ol.ph. & $\begin{array}{l}\text { Two size generations of skeletal forms; } \\
\text { larger ones displaying mottling and ex- } \\
\text { pansion cracks; some fringed by a sec. } \\
\text { extra fine grained titanomag. } \\
\text { larger: } 63 \mu \mathrm{m} \\
\text { smaller: } 5 \mu \mathrm{m}\end{array}$ & $\begin{array}{l}\text { Primary laths, partly formed like } \\
\text { titanomag. skeletals, some man- } \\
\text { tled by titanomag.; } 35 \mu \mathrm{m}\end{array}$ & $\begin{array}{l}\text { Rare small grains of pyrrhotite; } \\
1 \mu \mathrm{m}\end{array}$ & $\begin{array}{l}\text { Increasing content of iron } \\
\text { hydroxide; ilmenite is partly } \\
\text { replaced by iron hydrox. and } \\
\text { pyrite }\end{array}$ \\
\hline $17-1,46-55$ & pl.ol.cp.ph. & $\begin{array}{l}\text { Small skeletal forms; larger displaying, } \\
\text { maghemitization and sometimes tiny } \\
\text { fringes of hematite; } \\
12 \mu \mathrm{m} \text {-sub. }\end{array}$ & Usually small prim. laths; $15 \mu \mathrm{m}$ & $\begin{array}{l}\text { Small isometric pyrrhotite and } \\
\text { pyrite; some spherical structures of } \\
\text { original pyrite replaced by iron } \\
\text { hydroxide }\end{array}$ & $\begin{array}{l}\text { Rare large euhedral, homo- } \\
\text { geneous chromites, } 10-20 \mu \mathrm{m}, \\
\text { show a small seam of titanomag.; } \\
\text { seam is absent in the part lying in } \\
\text { plag. phenocrysts; iron hydroxide } \\
\text { in and around the displaced } \\
\text { olivines replaces titanomag. }\end{array}$ \\
\hline $21-1,112-123$ & $\begin{array}{l}\text { pl.ol.cpx. } \\
\text { phyric }\end{array}$ & $\begin{array}{l}\text { Subhedral to } s k e l e t a l \text { forms; larger ones } \\
\text { show mottling by maghemitization; } \\
15 \mu \mathrm{m}\end{array}$ & $\begin{array}{l}\text { Small prim. laths and two larger } \\
\text { ilmenohematite; } 20 \mu \mathrm{m}\end{array}$ & Considerable content of pyrrhotite & $\begin{array}{l}\text { One large porous chromite, } 60 \mu \mathrm{m} \text {, } \\
\text { partly rimmed by a fine seam of } \\
\text { titanomag; increasing content of } \\
\text { iron hydroxide in veinlets and } \\
\text { around the pyroxenes and olivines } \\
\end{array}$ \\
\hline $22-2,125-130$ & $\begin{array}{l}\text { pl.ol.cpx. } \\
\text { phyric }\end{array}$ & $\begin{array}{l}\text { Two generations; larger one anhedral, } \\
\text { smaller one skeletal; former are mottled; } \\
\text { near the skeletal margins lie tiny hema- } \\
\text { tite grains. Partly red-stained surround- } \\
\text { ing groundmass; } \\
\text { larger: } 12 \mu \mathrm{m} \\
\text { smaller: } 5 \mu \mathrm{m}\end{array}$ & $\begin{array}{l}\text { Larger grains, habit like titano- } \\
\text { mag. and small laths; all prim. } \\
\text { phases; } 10 \mu \mathrm{m}\end{array}$ & $\begin{array}{l}\text { Small roundish pyrrhotite, partly } \\
\text { enclosed by plag. phenocryst; } \\
1 \mu \mathrm{m}\end{array}$ & $\begin{array}{l}\text { Isometric, homogeneous chromite } \\
\text { grain, } 50 \mu \mathrm{m} \text {, sometimes rimmed } \\
\text { by a fine seam of titanomag.: } \\
\text { abundant iron hydroxide, partly } \\
\text { replacing titanomag. }\end{array}$ \\
\hline $23-1,137-142$ & $\begin{array}{l}\text { pl.ol.cpx. } \\
\text { phyric }\end{array}$ & $\begin{array}{l}\text { Small skeletal shapes; weak mottling and } \\
\text { expansion cracks, some fringed by fine- } \\
\text { granuled hematite; } 6 \mu \mathrm{m}\end{array}$ & Small prim. laths; $3 \mu \mathrm{m}$ & $\begin{array}{l}\text { Tiny roundish forms of indet. } \\
\text { grains; } 1 \mu \mathrm{m}\end{array}$ & $\begin{array}{l}\text { Large anhedral chromite grain } \\
\text { show a fine seam of titanomag.; } \\
50 \mu \mathrm{m}\end{array}$ \\
\hline $28-1,44-48$ & $\begin{array}{l}\text { pl.ol. } \\
\text { phyric }\end{array}$ & $\begin{array}{l}\text { Small skeletal grains, altered, mottled like } \\
23-1 ; 3 \mu \mathrm{m}\end{array}$ & Small prim. laths; $3 \mu \mathrm{m}$ & Too small for det.; $1 \mu \mathrm{m}$ & $\begin{array}{l}\text { Great content of iron hydroxide, } \\
\text { on basis of decomposition of } \\
\text { olivine, red staining of the adja- } \\
\text { cent groundmass; euhedral } \\
\text { chromite enclosed by plag. } \\
\text { phenocrysts; } 30 \mu \mathrm{m}\end{array}$ \\
\hline $31-1,125-128$ & $\begin{array}{l}\text { pl.ol.(cpx) } \\
\text { phyric }\end{array}$ & $\begin{array}{l}\text { Small skeletal grains; partly mottled by } \\
\text { maghemitization, partly fringed by fine- } \\
\text { grained hematite; } 5 \mu \mathrm{m}\end{array}$ & $\begin{array}{l}\text { Larger subhedral grains; another } \\
\text { more common phase composed } \\
\text { of small laths; } 7 \mu \mathrm{m}\end{array}$ & $\begin{array}{l}\text { Fine pyrrhotite grains surrounded } \\
\text { by plag.ph. }\end{array}$ & $\begin{array}{l}\text { Abundant content of iron } \\
\text { hydroxide lining veinlets and } \\
\text { vesicles; large homogeneous and } \\
\text { porous chromites; } 30 \mu \mathrm{m}\end{array}$ \\
\hline $32-1,140-150$ & $\begin{array}{l}\text { volcanic } \\
\text { breccia }\end{array}$ & $\begin{array}{l}\text { Two skeletal generations from different } \\
\text { grain size; smaller range from } 15 \mu \mathrm{m} \\
\text { down to the limit of visibility, larger } \\
\text { from } 150 \text { to } 30 \mu \mathrm{m} \text {; latter show mottling } \\
\text { and expan. cracks. Others contain iso- } \\
\text { lated coarse lamellae of ilmenite. High- } \\
\text { temperature oxidation followed by low- } \\
\text { temp. oxidation; } \\
\text { larger: } 130 \mu \mathrm{m} \\
\text { smaller: } 10 \mu \mathrm{m}\end{array}$ & $\begin{array}{l}\text { Prim. and secondary (lamellae) } \\
\text { phases; former are laths, mostly } \\
\text { mantled by titanomag., replaced } \\
\text { by iron hydroxide again. Smaller } \\
\text { laths displaying twin lam. and } \\
\text { partly parallel arrangement; } \\
60 \mu \mathrm{m}\end{array}$ & $\begin{array}{l}\text { Abundant pyrrhotite and pyrite, } \\
\text { partly as separated grains, partly as } \\
\text { multiphase of both }\end{array}$ & $\begin{array}{l}\text { Some euhedral grains of chromite; } \\
\text { homogeneous but slight mottling; } \\
50 \mu \mathrm{m}\end{array}$ \\
\hline
\end{tabular}


TABLE 1 - Continued

\begin{tabular}{|c|c|c|c|c|c|}
\hline $\begin{array}{l}\text { Sample } \\
\text { (Interval in } \\
\mathrm{cm})\end{array}$ & Lithology & Titanomagnetite & Ilmenite & Sulfides & Other Phases \\
\hline $33-1,70-77$ & $\begin{array}{l}\text { pl.ol. } \\
\text { phyric }\end{array}$ & $\begin{array}{l}\text { Skeletal shapes of very fine grain size; } \\
\text { expan. cracks and mottling by maghemi- } \\
\text { tization; } 10 \mu \mathrm{m} \text {-sub. }\end{array}$ & Very fine prim. laths; $10 \mu \mathrm{m}$-sub. & $\begin{array}{l}\text { Small pyrrhotite and less pyrite; } \\
5 \mu \mathrm{m}\end{array}$ & $\begin{array}{l}\text { Rare, homogeneous chromite } \\
\text { grains, partly rimmed by a tiny } \\
\text { seam of titanomag.; } 15 \mu \mathrm{m}\end{array}$ \\
\hline $49-1,77-84$ & $\begin{array}{l}\text { volcanic } \\
\text { breccia }\end{array}$ & $\begin{array}{l}\text { Two generations; smaller consists of } \\
\text { skeletal forms (in the glassy matrix), } \\
\text { larger are skelet. and fragments in the re- } \\
\text { crystallized areas. Expan. cracks. } \\
\text { larger: } 15 \mu \mathrm{m} \\
\text { smaller: } 5 \mu \mathrm{m}\end{array}$ & $\begin{array}{l}\text { Relatively high content; larger } \\
\text { show a rim of titanomag., another } \\
\text { are small laths and show texture } \\
\text { like titanomag. } \\
15 \mu \mathrm{m} \\
\text { tabular: } 40 \mu \mathrm{m}\end{array}$ & Rare, very small indet. grains & $\begin{array}{l}\text { Abundant content of iron } \\
\text { hydroxide in or around the } \\
\text { olivines and vesicles }\end{array}$ \\
\hline $52-1,45-50$ & $\begin{array}{l}\text { aphyric } \\
\text { fine gr. }\end{array}$ & $\begin{array}{l}\text { Extremely fine grained skeletal to } \\
\text { anhedral forms, displaying mottling more } \\
\text { reddish, partly colored int. reflections, } \\
\text { and tiny rims of maghemitization } \\
1 \mu \mathrm{m}\end{array}$ & $\begin{array}{l}\text { Tiny prim. laths and larger with } \\
\text { seams of titanomag. }\end{array}$ & $\begin{array}{l}\text { Extremely fine roundish indet. } \\
\text { grains; } 1 \mu \mathrm{m}\end{array}$ & $\begin{array}{l}\text { Iron hydroxide fills veinlets and } \\
\text { vesicles. }\end{array}$ \\
\hline $57-1,47-53$ & $\begin{array}{l}\text { aphyric } \\
\text { fine gr. }\end{array}$ & $\begin{array}{l}\text { Very fine skeletal to anhedral shapes, yet } \\
\text { without the reddish tints, as in } 52 \cdot 1 \text {; } \\
1 \mu \mathrm{m}\end{array}$ & Some prim. laths; $1 \mu \mathrm{m}$ & Rare small indet. grains; $1 \mu \mathrm{m}$ & \\
\hline $58-2,88-94$ & hyaloclastit. & & & $\begin{array}{l}\text { Some grains of pyrite enclosed by } \\
\text { plag. phenocr., partly intergrown } \\
\text { with pyrrhotite }\end{array}$ & 2 \\
\hline $61-1,140-150$ & aphyric & $\begin{array}{l}\text { Small skeletals, partly fringed by extreme- } \\
\text { ly fine grained hematite maghemite } \\
\text { appearances on the straits of the skeletals } \\
\text { (for example the axes or spurs of the } \\
\text { centers of the planes). Expan. cracks; } \\
20 \mu \text { m-sub. }\end{array}$ & $\begin{array}{l}\text { Small prim. laths, fringed likewise } \\
\text { by hematite; } 3 \mu \mathrm{m} \text {-sub. }\end{array}$ & $\begin{array}{l}\text { Some larger pyrrhotite and in the } \\
\text { majority, small, round, ind. } \\
\text { grains; } 5 \mu \mathrm{m}\end{array}$ & $\begin{array}{l}\text { Rare iron hydroxide, mostly in } \\
\text { vesicles }\end{array}$ \\
\hline $62-1,40-50$ & $\begin{array}{l}\text { aphyric, } \\
\text { fine-grained }\end{array}$ & $\begin{array}{l}\text { Very fine grained skeletal shapes with } \\
\text { slight reddish tints; expan. cracks and } \\
\text { fringes of fine hematite; } 10 \mu \mathrm{m} \text {-sub. }\end{array}$ & $\begin{array}{l}\text { Many small prim. laths, some } \\
\text { mantled by titanomag.; } 5 \mu \mathrm{m}\end{array}$ & $\begin{array}{l}\text { Fine grains of pyrite and finer ones } \\
\text { of pyrrhotite; } 1 \mu \mathrm{m}\end{array}$ & $\begin{array}{l}\text { Iron hydroxide lining vesicles and } \\
\text { replacing titanomag. under red } \\
\text { staining of the adjacent ground- } \\
\text { mass }\end{array}$ \\
\hline $63-1,0-10$ & $\begin{array}{l}\text { pl.ol.cpx. } \\
\text { doleritic }\end{array}$ & $\begin{array}{l}\text { Two generations of skeletals from differ- } \\
\text { ent grain size; smaller ones around the } \\
\text { pyroxene boundaries; the larger ones con- } \\
\text { tain a network of sec. ilmenite (lamelae) } \\
\text { displaying hematite exsolutions to the } \\
\text { phase boundaries of magnetite; occasional } \\
\text { fine fringes of ilemenite around the } \\
\text { grains. Some cracks filled by a red-brown } \\
\text { phase (less distinct anisotropy as } \\
\text { ilmenite); all grains show strong corrosion } \\
\text { and numerous expan. cracks. High-temp. } \\
\text { oxidation; } \\
\text { larger: } 150-30 \mu \mathrm{m} \\
\text { smaller: } 15 \mu \mathrm{m}-\text { sub. }\end{array}$ & $\begin{array}{l}\text { Large prim. laths, sometimes } \\
\text { mantled by titanomag. and } \\
\text { secondary phase in the titano- } \\
\text { mag.; } 70 \mu \mathrm{m} \text {-sub. }\end{array}$ & $\begin{array}{l}\text { Masses of small pyrite, pryyhotite. } \\
\text { and polyphases of these; } 20 \mu \mathrm{m}\end{array}$ & $\begin{array}{l}\text { Homogeneous, euhedral chromite; } \\
15 \mu \mathrm{m}\end{array}$ \\
\hline $64-4,115-122$ & $\begin{array}{l}\text { pl.ol.epx. } \\
\text { doler. }\end{array}$ & $\begin{array}{l}\text { Two generations like } 63-1 ; \\
\text { larger: } 150-40 \mu \mathrm{m} \text {-sub. } \\
\text { smaller: } 5 \mu \mathrm{m} \text {-sub. }\end{array}$ & $\begin{array}{l}\text { Like } 63-1 \text {; some of the grains } \\
\text { pseudomorph. to skeletal } \\
\text { titanomag. }\end{array}$ & $\begin{array}{l}\text { Like } 63-1 \text {; some spherical shapes } \\
\text { rimmed by small magnetite grains } \\
\text { contain pyrite-pryyhotite inter- } \\
\text { grown. } 15 \mu \mathrm{m}\end{array}$ & $\begin{array}{l}\text { Iron hydroxide fills spherical } \\
\text { structures }\end{array}$ \\
\hline $64-1,137-142$ & $\begin{array}{l}\text { pl.ol.cpx. } \\
\text { doler. }\end{array}$ & $\begin{array}{l}\text { Two generations like } 63-1 \text {; only single } \\
\text { exsolution lamellae of ilmenite; } \\
\text { larger: } 80-30 \mu \mathrm{m} \\
\text { smaller: } 3 \mu \mathrm{m}\end{array}$ & $\begin{array}{l}\text { Like } 63-1 \text {; rare larger ones of } \\
\text { tabular habit; } 60-15 \mu \mathrm{m}\end{array}$ & & \\
\hline $64-2,67-73$ & $\begin{array}{l}\text { pl.ol.cpx. } \\
\text { doler. }\end{array}$ & $\begin{array}{l}\text { Two generations like } 63-1 \text {; curiously only } \\
\text { few grains contain single ilmenite } \\
\text { lamellae. }\end{array}$ & Like 63-1 & $\begin{array}{l}\text { Content strongly decreased; rare } \\
\text { indet. grains }\end{array}$ & $\begin{array}{l}\text { Titanomag. partly replaced by } \\
\text { iron hydroxide }\end{array}$ \\
\hline $64-2,120-132$ & $\begin{array}{l}\text { pl.ol.cpx. } \\
\text { doler. }\end{array}$ & $\begin{array}{l}\text { Very fine grained skeletal shapes show } \\
\text { colored and a red-stained groundmass } \\
\text { about the grains, indications of strong } \\
\text { alteration. } \\
3 \mu \mathrm{m} \text {-sub. }\end{array}$ & Some small prim. laths; $5 \mu \mathrm{m}$-sub. & Tiny indet. grains, $1 \mu \mathrm{m}$ & \\
\hline $64-4,5-10$ & $\begin{array}{l}\text { aph. fine } \\
\text { grained }\end{array}$ & $\begin{array}{l}\text { Smail anhedral to skeletal forms, mostly } \\
\text { arranged between the plag. laths of the } \\
\text { groundmass; expan. cracks and mottling; } \\
10 \mu \mathrm{m} \text {-sub. }\end{array}$ & $\begin{array}{l}\text { Many prim. laths contain exsolu- } \\
\text { tions of hematite; } 10 \mu \mathrm{m} \text {-sub. }\end{array}$ & & $\begin{array}{l}\text { Some larger clusters of iron } \\
\text { hydroxide in amygdales, usually } \\
\text { lining veinlets and vesicles }\end{array}$ \\
\hline $66-2,42-48$ & $\begin{array}{l}\text { aph. fine- } \\
\text { grained }\end{array}$ & $\begin{array}{l}\text { Very fine grained skeletals; expan. cracks } \\
\text { and tiny white reflecting particles near } \\
\text { the margins; } 15 \mu \mathrm{m} \text {-sub. }\end{array}$ & Rare tiny prim. laths; $3 \mu \mathrm{m}$ & & Like $64-4$, lower content \\
\hline $14-6,37-43$ & $\begin{array}{l}\text { f. gr. ph. } \\
\text { basalt }\end{array}$ & $\begin{array}{l}\text { Very small grains show skeletal to sub- } \\
\text { hedral forms, partly rimmed by a fine } \\
\text { granuled hematite. Appear to be two } \\
\text { types of grains with a different color and } \\
\text { a diff. magnetic behavior. The gray-blue } \\
\text { ones have less magnetization than the } \\
\text { red-brown ones. The former are more } \\
\text { oxidized (maghemite) than the other; } \\
3 \mu \mathrm{m}\end{array}$ & No record & $\begin{array}{l}\text { Extremely fine isometric grains; no } \\
\text { det.; } 1 \mu \mathrm{m}\end{array}$ & $\begin{array}{l}\text { Rare large euhedral chromite } \\
\text { grains, partly porous and always } \\
\text { rimmed by a small seam of titano- } \\
\text { mag.; } 140 \mu \mathrm{m}\end{array}$ \\
\hline $15-1,115-123$ & $\begin{array}{l}\text { f. gr. pl. } \\
\text { ph. bas. }\end{array}$ & $\begin{array}{l}\text { Extremely fine grained subhedral forms; } \\
\text { otherwise like } 14-6 ; 2 \mu \mathrm{m}\end{array}$ & No record & $\begin{array}{l}\text { Some small grains of intergrown py- } \\
\text { rite. Chalcopyrite sometimes re- } \\
\text { placed by iron hydroxide from } \\
\text { margin; } 1 \mu \mathrm{m}\end{array}$ & $\begin{array}{l}\text { Iron hydroxide partly replaces } \\
\text { titanomag.; red staining of the } \\
\text { surrounding groundmass; rare } \\
\text { large homogeneous chromite; } \\
43 \mu \mathrm{m}\end{array}$ \\
\hline
\end{tabular}


TABLE 1 - Continued

\begin{tabular}{|c|c|c|c|c|c|}
\hline $\begin{array}{l}\text { Sample } \\
\text { (Interval in } \\
\mathrm{cm})\end{array}$ & Lithology & Titanomagnetite & llmenite & Sulfides & Other Phases \\
\hline $15-2,8-14$ & $\begin{array}{l}\text { f. gr. pl. } \\
\text { ph. bas. }\end{array}$ & Finer grain size than $15-1 ; 2 \mu \mathrm{m}$ & No record & Finer than $15-1$ & $\begin{array}{l}\text { Some euhedral microphenocrysts } \\
\text { of dense, homogeneous chromite; } \\
45 \mu \mathrm{m}\end{array}$ \\
\hline $15-4,72-78$ & $\begin{array}{l}\text { f. gr. pL } \\
\text { ph. bas. }\end{array}$ & $\begin{array}{l}\text { Very small skeletal to subhedral shapes } \\
\text { show different stages of oxidation. Some } \\
\text { contain expansion cracks, some fringed } \\
\text { by fine granuled hematite. Red staining } \\
\text { of the groundmass near the crystals; } \\
3 \mu \mathrm{m}\end{array}$ & Some primary laths, $2 \mu \mathrm{m}$ & $\begin{array}{l}\text { Roundish, drop-like grains; not } \\
\text { det.; } 1 \mu \mathrm{m}\end{array}$ & $\begin{array}{l}\text { A large euhedral homogeneous } \\
\text { chromite; content of iron hydrox- } \\
\text { ide slightly increasing; } 25 \mu \mathrm{m}\end{array}$ \\
\hline $15-4,53-111$ & $\begin{array}{l}\text { f. gr. pl. px. } \\
\text { ph. basalt }\end{array}$ & $\begin{array}{l}\text { Small skeletal grains of different oxida- } \\
\text { tion stages; } 15 \mu \mathrm{m} \text {-sub. }\end{array}$ & $\begin{array}{l}\text { Small laths and grains of a dis- } \\
\text { tinct tabular habit; some are re- } \\
\text { placed by hematite from margin. } \\
5 \mu \mathrm{m} \text {-sub. }\end{array}$ & Small roundish indet. grains; $3 \mu \mathrm{m}$ & $\begin{array}{l}\text { Iron hydroxide replaces titano- } \\
\text { mag., whereby the surrounding } \\
\text { groundmass is red-stained. }\end{array}$ \\
\hline $16-2,113-117$ & $\begin{array}{l}\text { f. pl. ph. } \\
\text { basalt }\end{array}$ & $\begin{array}{l}\text { Skeletal to subhedral forms; larger ones } \\
\text { mottled by low-temperature oxidation; } \\
5 \mu \mathrm{m}\end{array}$ & Rare fine primary laths; $5 \mu \mathrm{m}$ & $\begin{array}{l}\text { Tiny grains of pyrite and pyrtho- } \\
\text { tite; } 2 \mu \mathrm{m}\end{array}$ & $\begin{array}{l}\text { Larger, homogeneous euhedral } \\
\text { chromites show fine seams of } \\
\text { titanomag; decreasing content of } \\
\text { iron hydroxide; } 110 \mu \mathrm{m}\end{array}$ \\
\hline $16-4,76-85$ & $\begin{array}{l}\text { f. pl. gt. } \\
\text { ph. bas. }\end{array}$ & $\begin{array}{l}\text { Small skeletal to subhedral shapes; partly } \\
\text { rimmed by a fine granuled hematite, } \\
\text { partly containing expan. cracks; } 5 \mu \mathrm{m}\end{array}$ & $\begin{array}{l}\text { Few small laths of primary phase; } \\
3 \mu \mathrm{m}\end{array}$ & No det. fine grains; $1 \mu \mathrm{m}$ & Chromite like $16-2 ; 65 \mu \mathrm{m}$ \\
\hline $18-2,18-26$ & $\begin{array}{l}\text { extr. f. pl. } \\
\text { ph. basalt }\end{array}$ & Extremely fine grained; $1 \mu \mathrm{m}$ & Extr. fine gr.; $1 \mu \mathrm{m}$ & & $\begin{array}{l}\text { Microphenocrysts of chromite; } \\
30 \mu \mathrm{m}\end{array}$ \\
\hline $19-1,94-102$ & $\begin{array}{l}\text { f. gr. pl. } \\
\text { ph. bas. }\end{array}$ & $\begin{array}{l}\text { Small skeletal forms, mottled but only } \\
\text { little expan. cracks; some fringed by fine- } \\
\text { grained hematite; } 30 \mu \mathrm{m} \text {-sub. }\end{array}$ & Some prim. laths; $5 \mu \mathrm{m}$ & Fine isometric grains; $3 \mu \mathrm{m}$ & $\begin{array}{l}\text { Large euhedral, porous chromite; } \\
150 \mu \mathrm{m}\end{array}$ \\
\hline $22-3,95-107$ & $\begin{array}{l}\text { f. gr. pl. px. } \\
\text { ph. basalt }\end{array}$ & Extremely fine skeletal shapes; $1 \mu \mathrm{m}$ & No record & $\begin{array}{l}\text { Masses of tiny grains between the } \\
\text { plag. laths of the groundmass; } 1 \mu \mathrm{m}\end{array}$ & $\begin{array}{l}\text { Large euhedral, partly porous } \\
\text { chromite; } 50 \mu \mathrm{m}\end{array}$ \\
\hline $22-3,106-117$ & $\begin{array}{l}\text { fine gr. pl. } \\
\text { ph. basalt }\end{array}$ & Extremely small skeletal shapes; $1 \mu \mathrm{m}$ & Some prim. laths; $3 \mu \mathrm{m}$ & $\begin{array}{l}\text { Large grains enclosed by plag. } \\
\text { phenocrysts; corrosion of pyrr- } \\
\text { hotite by pyrite produces a kind of } \\
\text { network. }\end{array}$ & $\begin{array}{l}\text { Many large euhedral chromites } \\
\text { lie mainly near the pyrox. } \\
\text { phenocrysts; some contain inclus- } \\
\text { ions of groundmass; } 70 \mu \mathrm{m}\end{array}$ \\
\hline $22-3,117-118$ & $\begin{array}{l}\text { f. gr. pl. } \\
\text { px.ph. bas. }\end{array}$ & $\begin{array}{l}\text { Small skeletal forms; mottled and partly } \\
\text { fringed by fine granuled hematite; } 5 \mu \mathrm{m}\end{array}$ & Rare prim. laths; $5 \mu \mathrm{m}$ & Tiny roundish grans; $1 \mu \mathrm{m}$ & $\begin{array}{l}\text { A large euhedral porous chromite } \\
\text { enclosed by pyrox.; homogeneous } \\
\text { one lies in plag. phenocr.; } 100 \mu \mathrm{m} \\
\end{array}$ \\
\hline $22-4,77-87$ & $\begin{array}{l}\text { f. gr. pl. } \\
\text { ph. basait }\end{array}$ & Extremely small skeletal forms; $1 \mu \mathrm{m}$ & No record & $\begin{array}{l}\text { Increasing content pyrrh. ingrown } \\
\text { with pyrite }\end{array}$ & $\begin{array}{l}\text { Large euhedral chromites, partly } \\
\text { porous, partly homogeneous, but } \\
\text { all rimmed by a fine seam of titano } \\
\text { mag.; } 80 \mu \mathrm{m}\end{array}$ \\
\hline $24-2,87-98$ & $\begin{array}{l}\text { f. gr. pl. } \\
\text { ph. basalt }\end{array}$ & $\begin{array}{l}\text { Few large euhedral grains and masses of } \\
\text { small skeletal forms; some show a titano- } \\
\text { mag.-richer core; } 5 \mu \mathrm{m}\end{array}$ & Small prim. laths; $5 \mu \mathrm{m}$ & Very fine grain; $1 \mu \mathrm{m}$ & $\begin{array}{l}\text { Mostly small, homogeneous and a } \\
\text { large porous grain of chromite; in- } \\
\text { creasing content of iron hyd. near } \\
\text { the margins of pyroxenes and the } \\
\text { decomposed spinels; } 80-10 \mu \mathrm{m}\end{array}$ \\
\hline $24-3,16-24$ & $\begin{array}{l}\text { fine gr. pl. } \\
\text { ol. ph. basalt }\end{array}$ & $\begin{array}{l}\text { Small skeletal to subhedral forms show } \\
\text { varying oxidation stages and expan. } \\
\text { cracks, some rimmed by hematite } 2 \mu \mathrm{m}\end{array}$ & $\begin{array}{l}\text { Rare prim. laths, some of which } \\
\text { are mantled by titanomag. }\end{array}$ & $\begin{array}{l}\text { Small pyrrh., partly enclosed by } \\
\text { plag. phenocr.; } 1 \mu \mathrm{m}\end{array}$ & $\begin{array}{l}\text { Iron hydroxide fills vesicles and } \\
\text { cracks of pyroxene }\end{array}$ \\
\hline $24-3,75-80$ & $\begin{array}{l}\text { extr. f. gr. pl. } \\
\text { px. phy. bas. }\end{array}$ & $\begin{array}{l}\text { Extremely small skeletal forms, some- } \\
\text { times fringed by fine-granuled hematite; } \\
2 \mu \mathrm{m}\end{array}$ & No record & $\begin{array}{l}\text { Tiny roundish grains and a larger } \\
\text { grain of pyrrhotite }\end{array}$ & $\begin{array}{l}\text { Large subhedral chromite with a } \\
\text { tiny fringe of titanomag.; } \\
150-30 \mu \mathrm{m}\end{array}$ \\
\hline $25-1,84-93$ & $\begin{array}{l}\text { fine gr. pl. } \\
\text { px. phy. bas. }\end{array}$ & $\begin{array}{l}\text { Small euhedral to subhedral shapes, } \\
\text { sometimes fringed by tiny sulfide } \\
\text { grains, mottled; } 10 \mu \mathrm{m}\end{array}$ & Prim. laths; $10 \mu \mathrm{m}$ & $\begin{array}{l}\text { Small grains and clusters of } \\
\text { marcasite }\end{array}$ & \\
\hline
\end{tabular}

Note: sub = submicroscopic grain size; px. = pyroxene; phy. = phyric: bas = basalt: ol. = oitvune: apn. = aphyne

breccia zone within phyric basalt (Unit P-5) and in the doleritic intrusion (Unit P-4), larger titanomagnetites occur with exsolved ilmenite lamellae (secondary ilmenite) of oxidation class 3 or 4 (Plate 1, Figures 3, 4; Plate 2, Figure 1).

Myrmekitic intergrowths of titanomagnetite with ilmenite and with the silicate groundmass (Sample 395A-32-1, 140-150 cm) demonstrate partial leaching of titanomagnetites by hydrothermal solutions (Plate 2, Figure 2).

Samples 395A-63-1, 0-10 cm shows a more distinct leaching of titanomagnetite from the crystal margin to the first exsolution lamellae of ilmenite, so that the titanomagnetite grains now are also fringed by ilmenite. The more resistant ilmenite lamellae have been left beyond the original grain borders, and now protrude into the groundmass (Plate 1, Figure 4; Plate 2, Figure 1).

\section{Ilmenite}

Both primary and secondary ilmenite can be distinguished texturally. The former, commonly lath-shaped, occurs in all samples. In a few cases, ilmenite has a tabular habit and somewhat larger grain size. Frequently, grains are developed similar to the skeletal forms of titanomagnetite (Plate 2, Figures 3, 4, 5), making identification difficult, especially in unfavorable cross-sections. Ilmenite mantled by titanomagnetite is common. Occasionally, more altered laths contain minute lamellae of exsolved hematite. Secondary ilmenite intergrown with titanomagnetite (subsolidus exsolution lamellae) is a product of high-temperature oxidation. It occurs in Samples 395A-32-1, 140-150 cm; 395A-63-1, 0-10 cm; 395A-63-4, 115-122 cm; 395A$64-1,137-142 \mathrm{~cm}$; and 395A-64-2, 67-73 cm. 
TABLE 2

Mieroprobe Analyses of Iron-Titanium Oxides (wt \%)

\begin{tabular}{|c|c|c|c|c|c|c|c|}
\hline Unit & & & & & & & \\
\hline Lithology & $\begin{array}{c}\text { Pl. ol } \\
\text { ph. Basalt }\end{array}$ & $\begin{array}{c}\text { Pl. ol } \\
\text { ph. Basalt }\end{array}$ & $\begin{array}{c}\text { Volcanic } \\
\text { Breccia }\end{array}$ & & $\begin{array}{c}\text { Beginning } \\
\text { of Dolerite }\end{array}$ & Dolerite & \\
\hline $\begin{array}{c}\text { Sample } \\
\text { (Interval in } \\
\mathrm{cm} \text { ) }\end{array}$ & $\begin{array}{l}\text { 14-1, } \\
87-99\end{array}$ & $\begin{array}{c}14-2 \\
125-134\end{array}$ & $\begin{array}{c}32-1 \\
140-150\end{array}$ & F & $\begin{array}{c}61-1 \\
140-150\end{array}$ & $\begin{array}{l}63-1, \\
0-10\end{array}$ & E \\
\hline \multicolumn{8}{|c|}{ Spinel Phase } \\
\hline $\mathrm{MnO}$ & 0.33 & 0.32 & 0.30 & 0.28 & 0.33 & 0.29 & 0.30 \\
\hline $\mathrm{Al}_{2} \mathrm{O}_{3}$ & 1.47 & 1.19 & 1.46 & 7.95 & 1.70 & 1.41 & 1.47 \\
\hline $\mathrm{FeO}$ & 70.09 & 72.14 & 70.33 & 68.84 & 69.64 & 70.03 & 69.65 \\
\hline $\mathrm{TiO}_{2}$ & 23.91 & 23.40 & 23.09 & 19.74 & 21.96 & 21.34 & 21.36 \\
\hline $\mathrm{MgO}$ & 0.57 & 0.55 & 0.81 & 0.58 & 0.47 & 0.52 & 0.39 \\
\hline $\mathrm{Cr}_{2} \mathrm{O}_{3}$ & 0.02 & 0.02 & 0.03 & - & - & 0.03 & - \\
\hline Sum & 96.39 & 97.62 & 96.02 & 90.42 & 94.10 & 93.62 & 93.17 \\
\hline \multicolumn{8}{|c|}{ Spinel Formula } \\
\hline Mn & 0.011 & 0.011 & 0.010 & & 0.011 & 0.010 & 0.010 \\
\hline $\mathrm{Al}$ & 0.068 & 0.056 & 0.068 & & 0.080 & 0.067 & 0.069 \\
\hline $\mathrm{Fe}^{2+}$ & $\begin{array}{l}1.666 \\
\end{array}$ & 1.656 & $1.630^{\circ}$ & & $\begin{array}{l}1.620 \\
\text {. }\end{array}$ & 1.604 & $\begin{array}{l}.009 \\
1.613\end{array}$ \\
\hline $\mathrm{Fe}^{3+}$ & 0.511 & 0.545 & 0.556 & & 0.602 & 0.642 & 0.640 \\
\hline $\mathrm{Ti}$ & 0.710 & 0.699 & 0.688 & & 0.659 & 0.645 & 0.645 \\
\hline $\mathrm{Mg}$ & 0.033 & 0.032 & 0.048 & & 0.028 & 0.031 & 0.023 \\
\hline $\mathrm{Cr}$ & 0.001 & 0.001 & 0.001 & & - & 0.001 & - \\
\hline \multicolumn{8}{|c|}{$\begin{array}{l}\text { Recalculated Alalyses } \\
\text { Ilmenite Basis }\end{array}$} \\
\hline $\mathrm{Fe}_{2} \mathrm{O}_{3}$ & 36.37 & 38.19 & 37.78 & & 36.93 & 37.83 & 37.34 \\
\hline $\mathrm{FeO}$ & 37.36 & 37.78 & 36.34 & & 36.41 & 35.99 & 36.05 \\
\hline Total & 100.03 & 101.45 & 99.81 & & 97.80 & 97.41 & 96.91 \\
\hline \multicolumn{8}{|c|}{ Ulvospinel Basis } \\
\hline $\mathrm{Fe} 2 \mathrm{O}_{3}$ & 20.45 & 22.60 & 22.39 & & 22.31 & 23.62 & 23.12 \\
\hline $\mathrm{FeO}$ & 51.69 & 51.81 & 50.18 & & 49.57 & 48.78 & 48.85 \\
\hline Total & 98.44 & 99.89 & 98.26 & & 96.34 & 95.99 & 95.49 \\
\hline Mol\% Usp. & 68.4 & 66.1 & 65.2 & & 64.7 & 62.9 & 63.5 \\
\hline & & & & & & & $\mathrm{s}$ \\
\hline \multicolumn{8}{|c|}{ Rhombohedral Phase } \\
\hline $\mathrm{MnO}$ & 0.38 & 0.35 & 0.33 & & 0.28 & 0.26 & 0.47 \\
\hline $\mathrm{Al}_{2} \mathrm{O}_{3}$ & 0.20 & 0.16 & 0.16 & & 0.26 & -20 & 0.38 \\
\hline $\mathrm{FeO}$ & 46.23 & 50.66 & 48.13 & & 43.02 & 47.15 & 53.50 \\
\hline $\mathrm{TiO}_{2}$ & 51.13 & 49.13 & 49.86 & & 49.67 & 48.77 & 41.67 \\
\hline $\mathrm{MgO}$ & 0.50 & 0.61 & 0.93 & & 0.40 & 0.32 & 0.69 \\
\hline $\mathrm{Cr}_{2} \mathrm{O}_{3}$ & 0.04 & 0.01 & - & & - & - & - \\
\hline Sum & 98.48 & 100.92 & 99.41 & & 93.63 & 96.50 & 96.71 \\
\hline \multicolumn{8}{|c|}{ Mineral Formula } \\
\hline $\mathrm{Mn}$ & 0.008 & 0.007 & 0.007 & & 0.006 & 0.003 & 0.010 \\
\hline $\mathrm{Al}$ & 0.006 & 0.003 & 0.005 & & 0.008 & - & 0.072 \\
\hline $\mathrm{Fe}^{2+}$ & 0.948 & 0.912 & 0.918 & & 0.929 & 0.934 & 0.800 \\
\hline $\mathrm{Fe}^{3+}$ & 0.043 & 0.112 & 0.075 & & 0.929 & 0.110 & 0.373 \\
\hline $\mathrm{Ti}$ & 0.975 & 0.942 & 0.960 & & 0.950 & 0.945 & 0.837 \\
\hline $\mathrm{Mg}$ & 0.019 & 0.023 & 0.035 & & 0.015 & 0.008 & 0.027 \\
\hline $\mathrm{Cr}$ & 0.001 & 0.001 & - & & - & - & - \\
\hline \multicolumn{8}{|c|}{ Recalculated Analyses } \\
\hline $\mathrm{Fe} \mathrm{O}_{3}$ & 1.70 & 8.78 & 5.87 & & _- & 4.59 & 16.69 \\
\hline $\mathrm{FeO}$ & 44.70 & 42.76 & 42.84 & & $\overline{-}$ & 43.02 & $\begin{array}{l}10.07 \\
35.78\end{array}$ \\
\hline Total & 98.65 & 101.80 & 99.99 & & $=$ & 96.96 & 98.68 \\
\hline Mol\% & 1.9 & 8.6 & 5.9 & & - & 4.6 & 20.1 \\
\hline $\mathrm{R}_{2} \mathrm{O}_{3}$ & & & & & & & \\
\hline Temp. $\left({ }^{\circ} \mathrm{C}\right)$ & 810 & 1020 & 955 & & - & 910 & 1140 \\
\hline $\mathrm{fO}_{2}$ & $10^{-16.0}$ & $10^{-10.7}$ & $10^{-12.0}$ & & - & $10^{-12.9}$ & $10^{-8.2}$ \\
\hline
\end{tabular}
procedure according to Carmichael (1967).

\section{Iron Hydroxides}

These are abundant, but always in altered areas, veins and vesicles. They partly replace iron-titanium oxides.

\section{Sulfides}

Sulfides make up a widespread but volumetrically subordinate mineral phase in these rocks. Pyrite and pyrrhotite are the most common sulfides, and show different shapes. Globules are abundant (a result of immiscibility of silica and sulfide melt) and are usually fringed by a fine-grained, strongly magnetic cubic phase (Plate 3, Figures 1, 2). Some of these globules contain a core of pyrite, pyrrhotite, or both; othersbetter characterized as spherules-contain extremely fine grained iron hydroxide which has replaced the original sulfide. A microprobe analysis of a fringe grain of such a spherule (Sample 32-1, 140-150 cm) gives a titanomagnetite with approximately 20 wt. per cent
$\mathrm{TiO}_{2}$. This titanium content is not so greatly different from that of the bulk titanomagnetites that one can demonstrate two different origins of the iron-titanium oxides. As results from Leg 34 (Ade-Hall and Johnson, 1976 ) illustrate, extensive analyses are necessary to resolve such problems. Only in the dolerite sample (63-1, 0-10 $\mathrm{cm}$ ) do occasional marcasite nodules (Plate 3, Figure 3) occur. In this sample, a two-phased sulfide grain, consisting of exsolution lamellae of chalcopyrite in a pyrrhotite host, was formed.

\section{Chromium Spinel}

This mineral is rare, and appears as isolated microphenocrysts in the rocks. Two textural varieties can be discerned: a homogeneous type and a porous type. Both kinds are normally rimmed by titanomagnetite crystals. However, when completely surrounded by pyroxene or by plagioclase phenocrysts, the homogeneous grains have no such rim.

\section{Hole 396}

This hole cored a fairly uniform pillow basalt series. Variation in the textures of the iron-titanium oxides is correspondingly small. Mottled color, occasional volume-change cracks, and fringes of hematite indicate progressive alteration of the grains, whose last stage is typified by the diffusion of $\mathrm{Fe}$ into the surrounding groundmass. All these phenomena point to strong alteration during low-temperature oxidation. Ilmenite, here developed only as primary laths, is not present in all samples, and can be overlooked, because of the very small grain size $(<5 \mu \mathrm{m})$. Roundish sulfide grains (pyrite, pyrrhotite, or a mixed phase of both) are common. In part, pyrite and pyrrhotite are intergrown with chalcopyrite, which is replaced by iron hydroxide. Chromium spinel is a microphenocryst in nearly all rocks, and is texturally similar to chromium spinels, as in Hole 395A.

\section{CHEMICAL ANALYSES}

We analyzed pairs of coexisting titanomagnetite and primary ilmenite in five samples from three different basalt layers of Hole 395A (Table 2):

\begin{tabular}{ccr}
\hline Core-Section & $\begin{array}{c}\text { Interval } \\
(\mathrm{cm})\end{array}$ & Piece \\
\hline $14-1$ & $87-99$ & 12 \\
$14-2$ & $125-134$ & 9 \\
$32-1$ & $140-150$ & 7 \\
$61-1$ & $140-150$ & 2 \\
$63-1$ & $0-10$ & 1 \\
\hline
\end{tabular}

The minor elements $\mathrm{Al}$ and $\mathrm{Cr}$ show a slight preference for titanomagnetite, whereas $\mathrm{Mg}$ and $\mathrm{Mn}$ show a distinct preference for ilmenite. This is in good agreement with observations of Mathison (1976) on the Somerset Dam basic intrusion. The chemical analyses indicate significant compositional differences between primary and secondary ilmenite: $\mathrm{Fe}, \mathrm{Mn}, \mathrm{Al}$, and $\mathrm{Mg}$ 
prefer secondary ilmenite, and $\mathrm{Ti}$ prefers primary ilmenite.

Total iron, determined originally as $\mathrm{FeO}$ in the microprobe analyses, is expressed as $\mathrm{FeO}$ and $\mathrm{Fe}_{2} \mathrm{O}_{3}$, according to the recalculation procedure of Carmichael (1967). The temperature and oxygen fugacities corresponding to these compositions were determined from the $\mathrm{f}\left(\mathrm{O}_{2}\right)$-T curves of Buddington and Lindsley (1964). The average total for the spinel phase, computed on the basis of ulvospinel and ilmenite, respectively, should be close to 100 per cent, thus allowing one to check the quality of the analyses. But this check is restricted to compositions lying in the field between the ulvospinel-magnetite and the magnetite-ilmenite join. The higher the oxidation state of titanomagnetite, the greater a deviation from a total of 100 per cent should be expected. This is in good agreement with recalculated analyses of minerals seen under the petrographic microscope to be progressively more altered. Sample $395 \mathrm{~A}-14-2,125-134 \mathrm{~cm}$ is the least altered of all samples, and yields, from the Buddington and Lindsley curves. $\mathrm{T}=1020^{\circ} \mathrm{C}$ and $\mathrm{f}\left(\mathrm{O}_{2}\right)=10^{-10.7} \mathrm{~atm}-$ the crystallization conditions of titanomagnetite and primary ilmenite in equilibrium with each other and with the magma.

These $\mathrm{f}\left(\mathrm{O}_{2}\right)$-T conditions suggest that cooling of the basalt was too quick for equilibrium to develop. Opposed to this, the lower temperature and $\mathrm{f}\left(\mathrm{O}_{2}\right)$ data from samples 395A-32-1, 140-150, 395A-63-1, 0-10, and 395A-14-1, 87-99, demonstrate a much slower cooling of basalt here, such that the iron-titanium phase was always in a state of equilibrium. Above these low temperatures, fast cooling rates prevented equilibrium. I infer high-temperature oxidation for Sample 395A-63-1, 0-10, which has a relatively hematite-rich ilmenite phase.

\section{Optical and Magnetic Zoning}

The widespread halmyrolytic alteration of titanomagnetite in submarine basalts is of more than mere mineralogical interest. Ade-Hall and Johnson (1976) show that the cation deficiency, $z$, of titanomagnetite is strongly correlated with a variation of magnetic properties. One of these properties, the magnetization of the iron-titanium oxides, can be observed very well using the magnetic colloid (Plate 3, Figures 4, 5, 6; Plate 4, Figures 1, 2). Under reflected light, Sample 395A-14-2, $125-134 \mathrm{~cm}$ appears to have only homogeneous titanomagnetite, but the colloid test demonstrates magnetic zoning, a result of low-temperature oxidation. By contrast, in Sample 395A-61-1, 140-150 cm, there is a sharp separation between a light, nonmagnetic phase and a darker, strongly magnetic phase. Plate 4 , Figure 2 shows a microprobe scan over the different magnetic areas of an oxidized titanomagnetite (titanomaghemite). The microprobe analyses of these different portions are given in Table 3 . The data demonstrate a slight preference of $\mathrm{Al}, \mathrm{Mg}$, and $\mathrm{Fe}$ for the strongly magnetic phase, and $\mathrm{Ti}$ and $\mathrm{Mn}$ to the lighter, nonmagnetic phase. All these investigated grains are strongly maghemitized, as the cation deficiency of the borders and the cores indicate. The cation deficiency is real, and is not a result of error in the microprobe analyses.

\section{SUMMARY AND DISCUSSION}

In all the investigated samples, titanomagnetite is the dominant phase; ilmenite is subordinate and sulfides much less abundant. Microscopic observations show that the fine-grained, rapidly cooled basalts are characterized by fine skeletal to anhedral forms of titanomagnetite. The frequent volume-change cracks and of mottling indicate low-temperature oxidation of the rocks. This is a common appearance of titanomagnetities in submarine basalts in ocean floor basalts (AdeHall and Johnson, 1976; Petersen et al., in press). In contrast to subaerial basalts, high-temperature oxidation of titanomagnetite seems to be rare in ocean floor basalts, and appears to occur only in the centers of massive flows and sills (Watkins et al., 1967; Gromme et al., 1969). In good agreement with this inference, high-temperature oxidation of titanomagnetite (class 3 or 4) was found in the dolerite intrusion. Microprobe analyses of an "exsolved" titanomagnetite from the dolerite give $\mathrm{f}\left(\mathrm{O}_{2}\right)$-T values which are too high to represent crystallization conditions. The values of $\mathrm{T}=$ $1020^{\circ} \mathrm{C}$ and $\mathrm{f}\left(\mathrm{O}_{2}\right)=10^{-11}$ atm obtained from Sample $395 \mathrm{~A}-14-2,125-134 \mathrm{~cm}$, are similar to results from Leg 34 (Mazullo et al., 1976), and may demonstrate more typical equilibrium conditions during the crystallization of iron-titanium oxides.

The preliminary results of optical and magnetic zoning of low-temperature-oxidized titanomagnetites are similar to those of Prevot (1968).

\section{ACKNOWLEDGMENTS}

The author wishes to acknowledge Prof. Dr. D. D. Klemm and Dr. N. Petersen for stimulating discussion during the course of this research. J. Burri provided helpful assistance in the microprobe laboratory of the University of LausanneDorigny. The financial support of the Deutsche Forschungsgemeinschaft is gratefully acknowledged.

\section{REFERENCES}

Ade-Hall, J. M. and Johnson, H. P., 1976. Petrography of opaque minerals, Leg 34. In Hart, S. R., Yeats, R. S., et al., Initial Reports of the Deep Sea Drilling Project, v. 34: Washington (U.S. Government Printing Office), p. 349362.

Buddington, A. F. and Lindsley, D. H., 1964. Iron-titanium oxide minerals and synthetic equivalents, J. Petrol., v. 5, p. 310-375.

Carmichael, I. S. E., 1967. The iron-titanium oxides of salic volcanic rocks and their associated ferromagnesian silicates, Contrib. Mineral. Petrol., v. 14, p. 36-44.

Grommé, C. S., Wright, T. L., and Peck, D. L., 1969. Magnetic properties and oxidation of iron-titanium oxide minerals in Alae and Makaopuhi lava lakes, Hawaii, J. Geophys. Res., v. 74, p. 5277-5293.

Mathison, C. I., 1975. Magnetites and ilmenites in the Somerset Dam layered basic intrusion, southeastern Queensland, Lithos, v. 8, p. 93-111. 
TABLE 3

Microprobe Analyses of Iron-Titanium Oxides Showing Optical and Magnetic Zoning (wt. \%)

\begin{tabular}{|c|c|c|c|c|c|c|c|c|c|c|c|c|c|c|c|c|c|c|}
\hline \multirow{2}{*}{$\begin{array}{c}\text { Sample } \\
\text { (Interval in cm) }\end{array}$} & \multicolumn{3}{|c|}{$\begin{array}{c}14-2 \\
125-134 \\
\end{array}$} & \multicolumn{2}{|c|}{$\begin{array}{c}61-1, \\
140-150 \\
\end{array}$} & \multicolumn{2}{|c|}{$\begin{array}{c}61-1, \\
140-150 \\
\end{array}$} & \multicolumn{11}{|c|}{$\begin{array}{c}32-1, \\
140-150\end{array}$} \\
\hline & 1 & 2 & 3 & 1 & 2 & $1^{*}$ & $2^{*}$ & 1 & 2 & 3 & 4 & 5 & 6 & 7 & 8 & 9 & 10 & 11 \\
\hline $\begin{array}{l}\mathrm{MnO} \\
\mathrm{Al}_{2} \mathrm{O}_{3} \\
\mathrm{FeO} \\
\mathrm{TiO} \\
\mathrm{MgO}^{2} \\
\mathrm{Cr}_{2} \mathrm{O}_{3} \\
\mathrm{Sum}\end{array}$ & $\begin{array}{r}0.31 \\
1.31 \\
70.92 \\
22.42 \\
0.55 \\
- \\
95.51\end{array}$ & $\begin{array}{r}0.30 \\
1.81 \\
76.60 \\
21.55 \\
0.60 \\
- \\
100.86\end{array}$ & $\begin{array}{r}0.30 \\
1.51 \\
75.91 \\
21.21 \\
0.66 \\
- \\
99.59\end{array}$ & $\begin{array}{r}0.35 \\
1.81 \\
67.32 \\
22.60 \\
0.52 \\
- \\
92.60\end{array}$ & $\begin{array}{r}0.36 \\
1.69 \\
68.79 \\
21.96 \\
0.54 \\
- \\
93.34\end{array}$ & $\begin{array}{r}0.30 \\
1.38 \\
68.03 \\
22.46 \\
0.40 \\
- \\
92.56\end{array}$ & $\begin{array}{r}0.30 \\
1.91 \\
70.41 \\
20.80 \\
0.40 \\
0.01 \\
93.83\end{array}$ & $\begin{array}{r}0.30 \\
1.44 \\
67.56 \\
22.65 \\
0.91 \\
- \\
92.86\end{array}$ & $\begin{array}{r}0.26 \\
1.56 \\
69.97 \\
23.05 \\
0.79 \\
0.08 \\
95.70\end{array}$ & $\begin{array}{r}0.27 \\
1.47 \\
71.77 \\
22.57 \\
0.85 \\
- \\
96.93\end{array}$ & $\begin{array}{r}0.30 \\
1.65 \\
72.62 \\
22.42 \\
0.75 \\
- \\
97.74\end{array}$ & $\begin{array}{r}0.28 \\
1.56 \\
72.37 \\
22.42 \\
0.80 \\
- \\
97.43\end{array}$ & $\begin{array}{r}0.26 \\
1.64 \\
72.63 \\
22.37 \\
0.82 \\
- \\
97.72\end{array}$ & $\begin{array}{r}0.31 \\
1.66 \\
71.93 \\
22.76 \\
0.88 \\
0.07 \\
97.60\end{array}$ & $\begin{array}{r}0.32 \\
1.73 \\
71.37 \\
22.39 \\
0.84 \\
0.06 \\
96.72\end{array}$ & $\begin{array}{r}0.28 \\
1.70 \\
70.01 \\
22.89 \\
0.78 \\
- \\
95.66\end{array}$ & $\begin{array}{r}0.29 \\
1.72 \\
69.15 \\
22.93 \\
0.82 \\
- \\
94.90\end{array}$ & $\begin{array}{r}0.31 \\
1.74 \\
68.85 \\
23.00 \\
0.76 \\
- \\
94.66\end{array}$ \\
\hline \multicolumn{19}{|l|}{ Spinel Formula } \\
\hline $\begin{array}{l}\mathrm{Mn} \\
\mathrm{Al}_{2+} \\
\mathrm{Fe}_{3+} \\
\mathrm{Fe} \\
\mathrm{Ti} \\
\mathrm{Mg} \\
\mathrm{Cr}\end{array}$ & $\begin{array}{l}0.010 \\
0.062 \\
1.630 \\
0.592 \\
0.673 \\
0.033 \\
-\end{array}$ & $\begin{array}{l}0.010 \\
0.085 \\
1.601 \\
0.621 \\
0.647 \\
0.036 \\
-\end{array}$ & $\begin{array}{l}0.010 \\
0.071 \\
1.591 \\
0.649 \\
0.640 \\
0.039 \\
-\end{array}$ & $\begin{array}{l}0.012 \\
0.085 \\
1.632 \\
0.565 \\
0.675 \\
0.031 \\
-\end{array}$ & $\begin{array}{l}0.012 \\
0.079 \\
1.615 \\
0.603 \\
0.659 \\
0.032 \\
-\quad\end{array}$ & $\begin{array}{l}0.010 \\
0.065 \\
1.641 \\
0.585 \\
0.675 \\
0.024 \\
-\end{array}$ & $\begin{array}{l}0.010 \\
0.090 \\
1.594 \\
0.653 \\
0.628 \\
0.047 \\
-\quad\end{array}$ & $\begin{array}{l}0.010 \\
0.067 \\
1.613 \\
0.579 \\
0.677 \\
0.054 \\
-\end{array}$ & $\begin{array}{l}0.009 \\
0.073 \\
1.630 \\
0.553 \\
0.686 \\
0.047 \\
0.002\end{array}$ & $\begin{array}{l}0.009 \\
0.069 \\
1.616 \\
0.581 \\
0.675 \\
0.050 \\
-\end{array}$ & $\begin{array}{l}0.010 \\
0.077 \\
1.616 \\
0.583 \\
0.670 \\
0.044 \\
-\end{array}$ & $\begin{array}{l}0.009 \\
0.073 \\
1.614 \\
0.587 \\
0.670 \\
0.047 \\
-\end{array}$ & $\begin{array}{l}0.009 \\
0.077 \\
1.611 \\
0.585 \\
0.669 \\
0.049 \\
-\end{array}$ & $\begin{array}{l}0.010 \\
0.077 \\
1.611 \\
0.565 \\
0.678 \\
0.052 \\
0.002\end{array}$ & $\begin{array}{l}0.011 \\
0.081 \\
1.607 \\
0.581 \\
0.668 \\
0.050 \\
0.002\end{array}$ & $\begin{array}{l}0.009 \\
0.079 \\
1.627 \\
0.557 \\
0.682 \\
0.046 \\
-\end{array}$ & $\begin{array}{l}0.010 \\
0.080 \\
1.621 \\
0.562 \\
0.679 \\
0.048 \\
-\quad\end{array}$ & $\begin{array}{l}0.010 \\
0.081 \\
1.629 \\
0.551 \\
0.684 \\
0.045 \\
-\end{array}$ \\
\hline \multicolumn{19}{|c|}{$\begin{array}{c}\text { Recalculated Analyses } \\
\text { Ilmenite Basis }\end{array}$} \\
\hline $\begin{array}{l}\mathrm{Fe}_{2} \mathrm{O}_{3} \\
\mathrm{FeO} \\
\text { Total }\end{array}$ & $\begin{array}{l}37.88 \\
36.84 \\
99.31\end{array}$ & $\begin{array}{r}42.46 \\
38.39 \\
105.10\end{array}$ & $\begin{array}{r}42.42 \\
37.74 \\
103.84\end{array}$ & $\begin{array}{l}34.82 \\
35.99 \\
96.02\end{array}$ & $\begin{array}{l}36.43 \\
36.01 \\
96.99\end{array}$ & $\begin{array}{l}35.46 \\
36.13 \\
96.12\end{array}$ & $\begin{array}{l}38.06 \\
36.17 \\
97.65\end{array}$ & $\begin{array}{l}35.64 \\
35.49 \\
96.43\end{array}$ & $\begin{array}{l}36.87 \\
36.79 \\
99.39\end{array}$ & $\begin{array}{r}38.69 \\
36.95 \\
100.80\end{array}$ & $\begin{array}{r}39.22 \\
37.33 \\
101.67\end{array}$ & $\begin{array}{r}39.13 \\
37.16 \\
101.35\end{array}$ & $\begin{array}{r}39.32 \\
37.24 \\
101.65\end{array}$ & $\begin{array}{r}38.63 \\
37.17 \\
101.47\end{array}$ & $\begin{array}{r}38.38 \\
36.63 \\
100.56\end{array}$ & $\begin{array}{l}36.97 \\
36.75 \\
99.37\end{array}$ & $\begin{array}{l}36.36 \\
36.44 \\
98.55\end{array}$ & $\begin{array}{l}36.01 \\
36.45 \\
98.27\end{array}$ \\
\hline \multicolumn{19}{|l|}{ Ulvospinel Basis } \\
\hline $\begin{array}{l}\mathrm{Fe}_{2} \mathrm{O}_{3} \\
\mathrm{FeO} \\
\text { Total } \\
\text { Mol \% Usp. }\end{array}$ & $\begin{array}{l}22.94 \\
50.28 \\
97.81 \\
64.7\end{array}$ & $\begin{array}{c}28.11 \\
51.31 \\
103.68 \\
58.8\end{array}$ & $\begin{array}{r}28.28 \\
50.46 \\
102.42 \\
58.4\end{array}$ & $\begin{array}{l}19.73 \\
49.57 \\
94.58 \\
67.9\end{array}$ & $\begin{array}{l}21.80 \\
49.17 \\
95.52 \\
65.1\end{array}$ & $\begin{array}{l}20.51 \\
49.58 \\
94.62 \\
67.3\end{array}$ & $\begin{array}{l}24.20 \\
48.63 \\
96.25 \\
61.6\end{array}$ & $\begin{array}{l}20.55 \\
49.07 \\
94.92 \\
66.9\end{array}$ & $\begin{array}{l}21.52 \\
50.61 \\
97.86 \\
66.4\end{array}$ & $\begin{array}{l}23.65 \\
50.49 \\
99.30 \\
63.8\end{array}$ & $\begin{array}{c}24.28 \\
50.77 \\
100.17 \\
63.1\end{array}$ & $\begin{array}{l}24.19 \\
50.60 \\
99.85 \\
63.2\end{array}$ & $\begin{array}{c}24.42 \\
50.66 \\
100.17 \\
62.9\end{array}$ & $\begin{array}{l}23.46 \\
50.82 \\
99.95 \\
64.0\end{array}$ & $\begin{array}{l}23.47 \\
50.26 \\
99.08 \\
63.7\end{array}$ & $\begin{array}{l}21.72 \\
50.47 \\
97.84 \\
66.0\end{array}$ & $\begin{array}{l}21.08 \\
50.18 \\
97.01 \\
66.6\end{array}$ & $\begin{array}{l}20.69 \\
50.23 \\
96.73 \\
67.1\end{array}$ \\
\hline
\end{tabular}


Mazullo, L. J. and Bence, A. E., 1976. Abyssal tholeiites from DSDP Leg 34: The Nazca Plate, J. Geophys. Res., v. 81, p. 4327-4351.

Petersen N., Bleil, U. and Eisenach, P., in press. Rock- and palaeomagnetism of Leg 43 basalts. In Tucholke, B., Vogt, P., et al., Initial Reports of the Deep Sea Drilling Project, v. 43, Washington (U.S. Government Printing Office).
Prévot, M., Rémond, G. and Caye, R., 1968. Etude de la transformation d'une titanomagnétite en titanomaghémite dans une roche volcanique, Bull. Soc. France Mineral. Cristallogr., v. 91, p. 65-74.

Watkins, N. D. and Haggerty, S. E., 1967. Primary oxidation variation and petrogenesis in a single lava, Contrib. Mineral. Petrol., v. 15, p. 251-271. 



\section{PLATE 1}

Figure 1 Microphenocryst of a large porous chromium spinel shows limited development of a tiny seam of titanomagnetite.

Sample 395-18-2, 35-37 cm.

Figure 2 Microphenocryst of a large homogeneous chromium spinel, partly fringed by titanomagnetite. Sample 395A-17-1, 69-75 cm.

Figure 3 Titanomagnetite of skeletal to anhedral form shows some secondary ilmenite lamellae and limited development of volume-change cracks, partly filled with sulfide phase.

Sample 395A-63-1, 0-10 cm.

Figure $4 \quad$ High deuteric oxidation state of an exsolved titanomagnetite grain; partial leaching of titanomagnetite produces a ilmenite fringe around the grain (with colloid).

Sample 395A-63-1, 0-10 cm. 


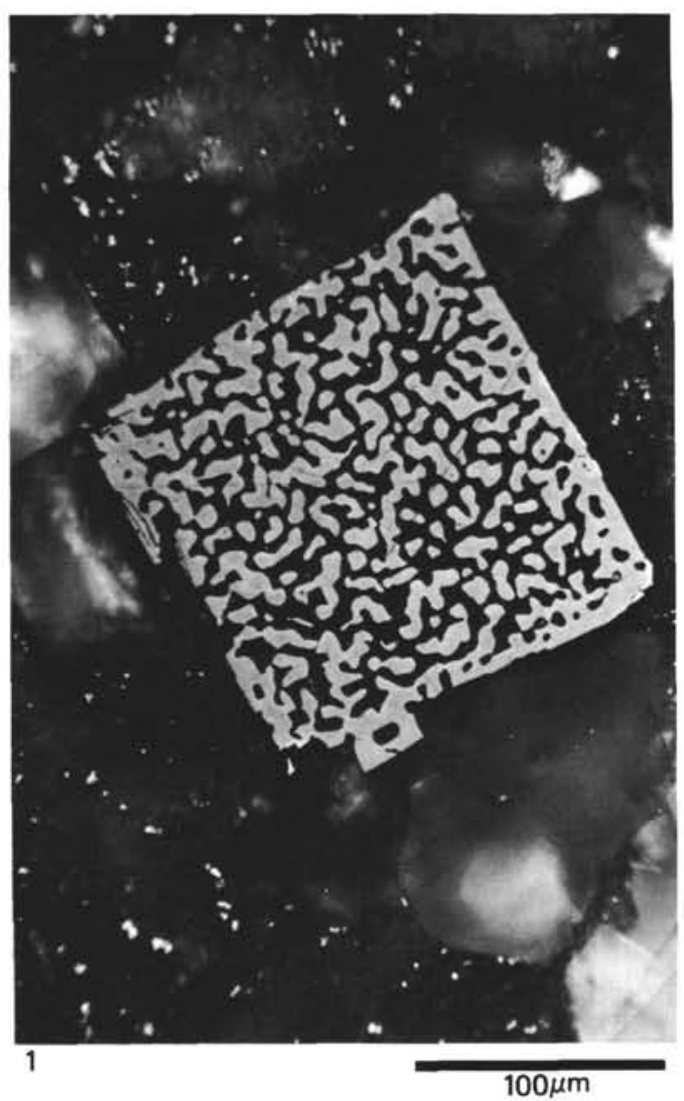

\section{PLATE 1}
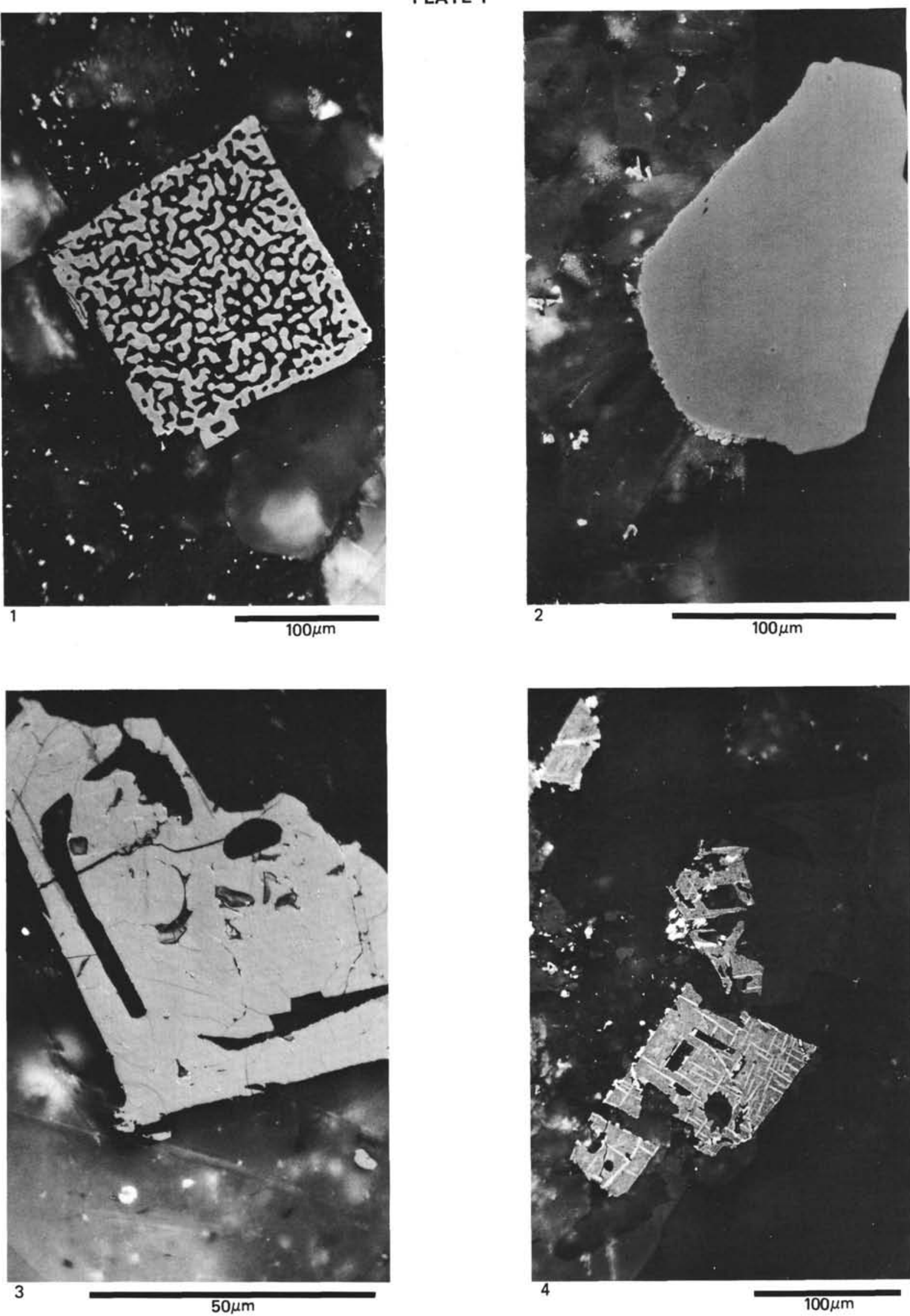
PLATE 2

Figure 1 High oxidation state of a titanomagnetite; the more resistant ilmenite lamellae spread beyond the grain borders into the silica groundmass by progressive leaching of the titanomagnetite phase. The white reflecting phase is a pyrrhotite grain. Colloid.

Sample 395A-63-1, 0-10 cm.

Figure 2 Myrmekitic intergrowth of ilmenite, silica groundmass, and titanomagnetite; small relicts of titanomagnetite in the worm-shaped portions demonstrate the replacement of titanomagnetite phase by silica groundmass.

Sample 395A-32-1, 140-150 cm.

Figure $3 \quad$ Ilmenite fringed by tiny sulfide grains formed similar to titanomagnetite skeletals; colloid shows different magnetization in the skeletals of titanomagnetite.

Sample 395A-14-2, 125-134 cm.

Figure 4 Ilmenite formed as skeletal titanomagnetite have been detected by magnetic colloid.

Sample 395A-14-1, 87-99 cm.

Figure 5 Skeletal ilmenite, formed similar to titanomagnetite skeletals, is partly mantled by titanomagnetite.

Sample 395A-14-2, 125-134 cm. 


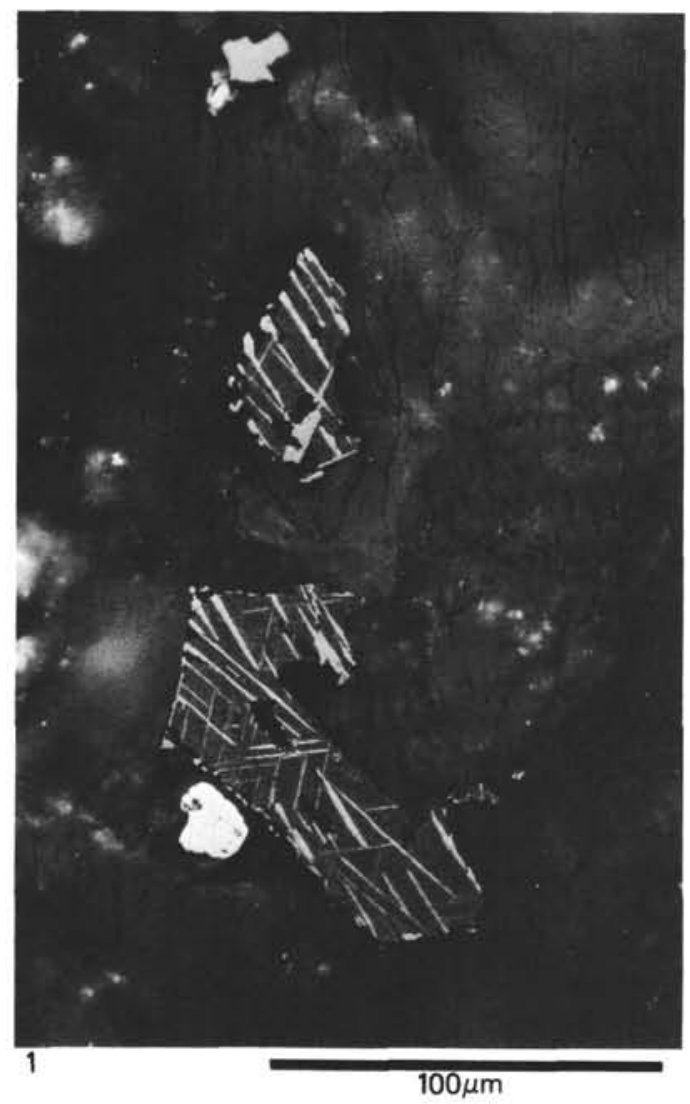

PLATE 2
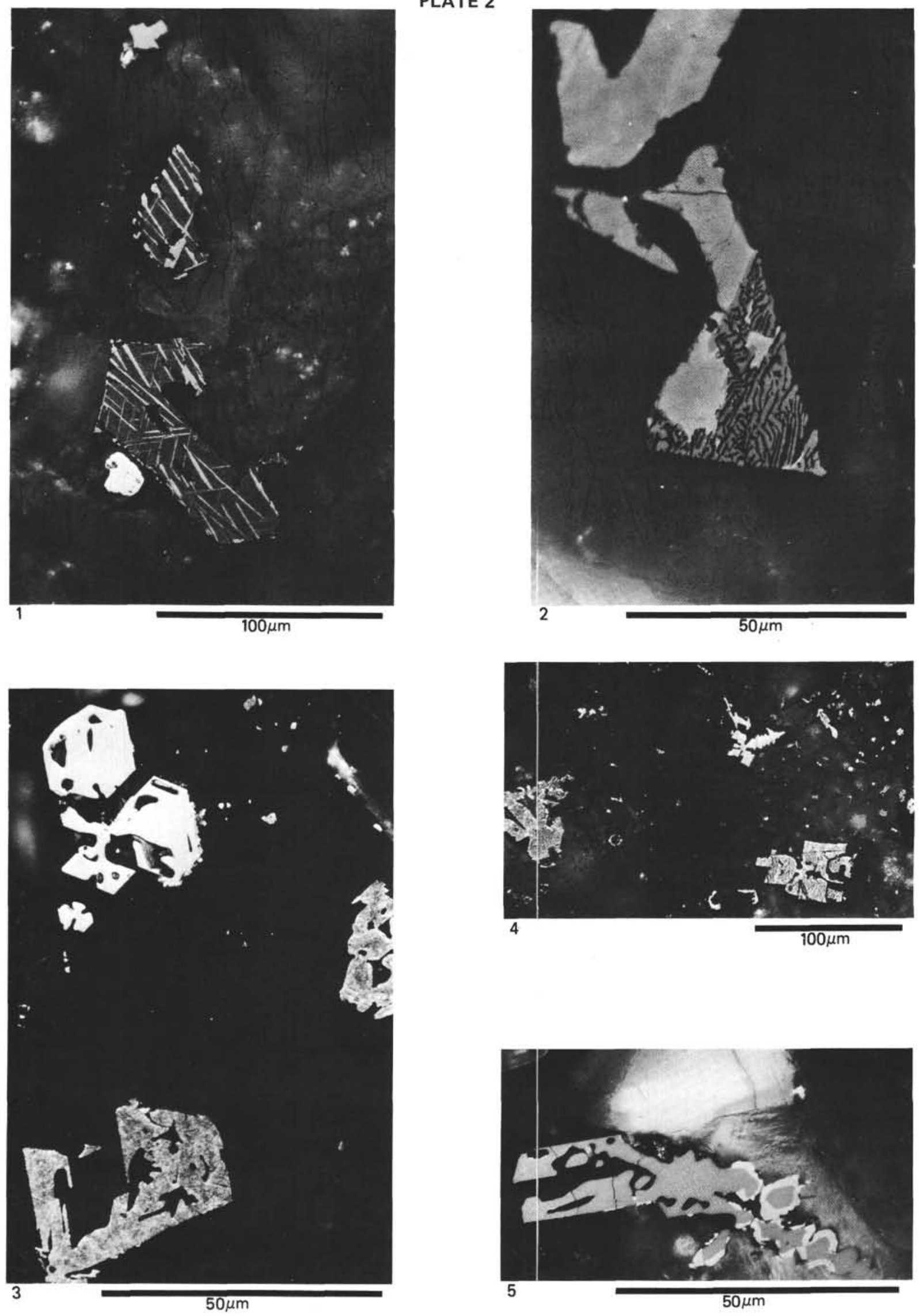


\section{PLATE 3}

Figure 1 Small sulfide globules lie near the skeletal titanomagnetite.

Sample 395A-14-2, 125-134 cm.

Figure 2 Spherule of an original pyrite, now are replaced by iron hyroxide; spherule is rimmed by tiny titanomagnetite grains (see microprobe analysis, Table 2 F).

Sample 395A-63-4, 137-142 cm.

Figure 3 Markasite nodule, crossed nicols. Sample 395A$63-1,0-10 \mathrm{~cm}$.

Figure 4 An optical homogeneous titanomagnetite shows magnetic zoning using the colloid test. Analyses points 1-3, see Table 3 .

Sample 395A-14-2, 125-134 cm.

Figure 5 Shows a sharp separation into a light phase and a darker reddish brown phase.

Sample 395A-61-1, 140-150 cm.

Figure 6 Colloid test demonstrates the strong magnetization in the darker phase of the same grain. Analyses points 1-2, see Table 3.

Sample 395A-61-1, 140-150 cm. 
PLATE 3
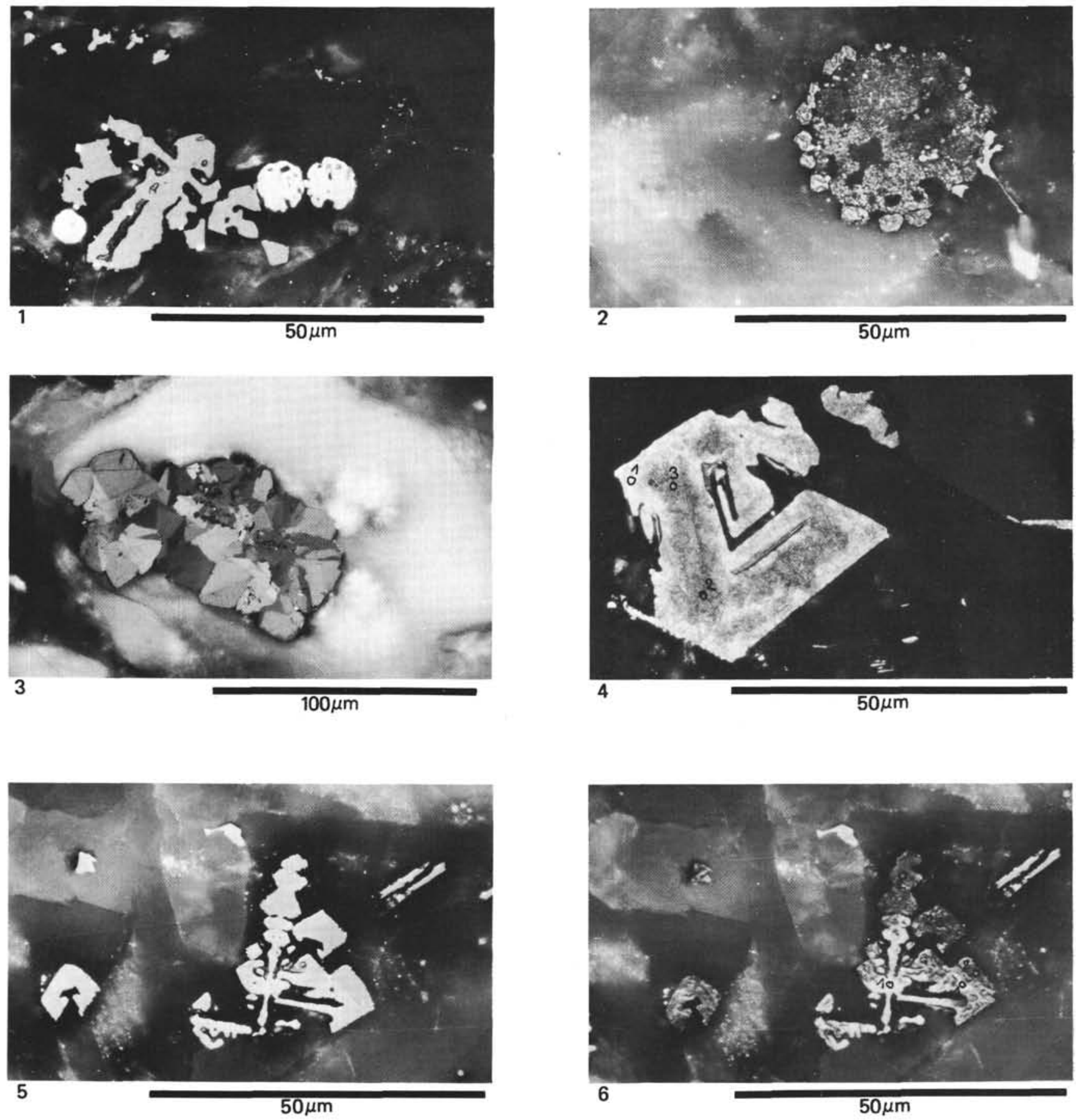
PLATE 4

Figure 1 Colloid shows different magnetization in a skeletal titanomagnetite (snowstar). Analyses points $1 *, 2 *$, see Table 3 .

Sample 395A-61-1, 140-150 cm.

Figure 2 Colloid demonstrates magnetic zoning resulting from low-temperature oxidation. A microprobe scan (analyses points 1-11) gives the variation in the chemical composition from the more maghemitized margin to the less maghemitized and also strong magnetic core. A spherule filled by iron hydroxide is partially rimmed by tiny titanomagnetite grains.

Sample 395A-32-1, 140-150 cm. 

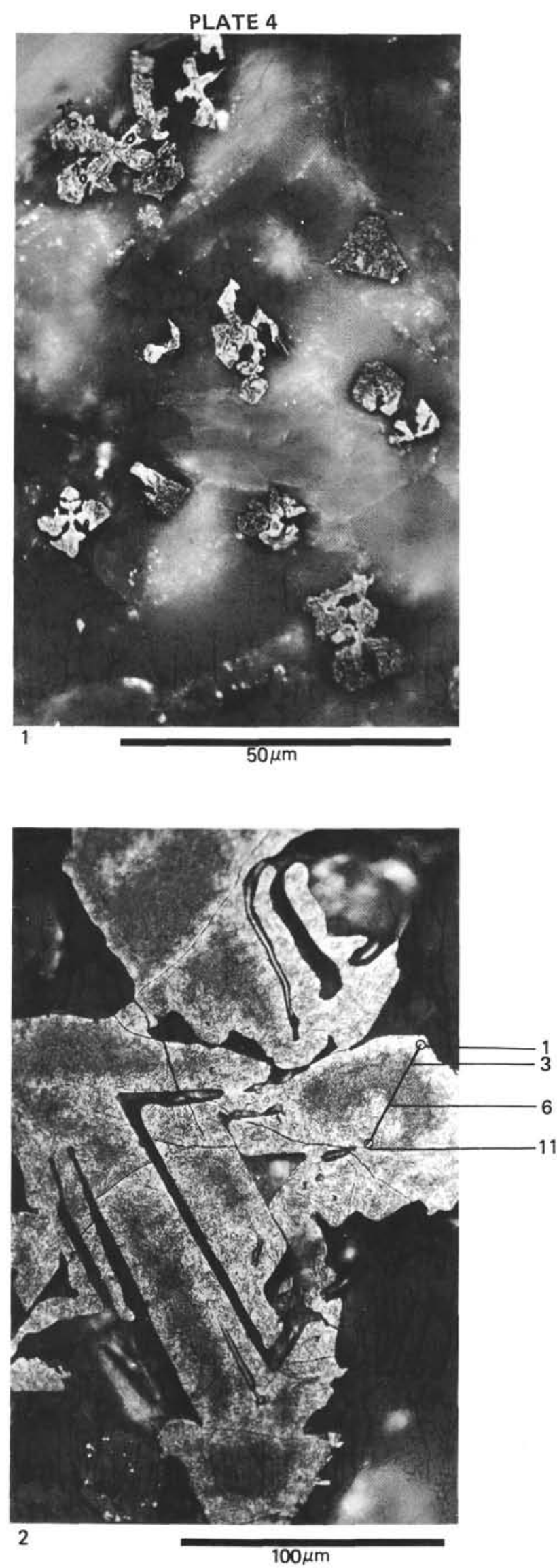